\title{
Accountability for the hidden codes toward a better user experience
}

\author{
Case study of HRsimple communication design for web accessibility and SEO
}

\author{
Justin M. Grant \\ American Chamber of Commerce \\ Resources, hrsimple.com, Chicago, \\ Illinois \\ jmgrant702@gmail.com
}

\author{
Gabrielle K. Sumilang \\ University of California, Los Angeles \\ gsumilang@ucla.edu
}

\author{
Michael Providenti \\ NKU, Highland Heights, Kentucky \\ providenti@nku.edu
}

\author{
Deborah Reichler \\ NKU, Highland Heights, Kentucky \\ reichler@nku.edu
}

\author{
Sookyung Cho* \\ University of California, Los Angeles \\ sookie@g.ucla.edu
}

\begin{abstract}
Search engine optimization and web accessibility for private industry are the key components to our research methods and examination of user experience through hidden code. Hidden code herein refers to frontend code such as structural HTML5 including landmark elements, WAI-ARIA, and CSS styling. In highlighting the relevance of web accessibility to increased search engine exposure, better web development practices, and overall more holistic user experience, this research aims to advocate for the importance of accountability in design practices to business stakeholders and industry perspectives. $<\mathrm{fn}$ id="fn3" fn-type="other" >
\end{abstract}

\section{CCS CONCEPTS}

- Human-centered computing $\rightarrow$ Accessibility; Empirical studies in accessibility.

\section{KEYWORDS}

Web Accessibility, Search Engine Optimization, User Experience

\section{ACM Reference Format:}

Justin M. Grant, Gabrielle K. Sumilang, Michael Providenti, Deborah Reichler, and Sookyung Cho. 2021. Accountability for the hidden codes toward a better user experience: Case study of HRsimple communication design for web accessibility and SEO. In The 39th ACM International Conference on Design of Communication (SIGDOC '21), October 12-14, 2021, Virtual Event, USA. ACM, New York, NY, USA, 4 pages. https://doi.org/10.1145/3472714.3473664

\footnotetext{
* Permission to make digital or hard copies of part or all of this work for personal or classroom use is granted without fee provided that copies are not made or distributed for profit or commercial advantage and that copies bear this notice and the full citation on the first page. Copyrights for third-party components of this work must be honored. For all other uses, contact the Owner/Author.
}

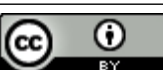

This work is licensed under a Creative Commons Attribution International 4.0 License.

SIGDOC '21, October 12-14, 2021, Virtual Event, USA

(C) 2021 Copyright held by the owner/author(s)

ACM ISBN 978-1-4503-8628-9/21/10.

https://doi.org/10.1145/3472714.3473664

\section{INTRODUCTION}

Despite being an important facet of holistic user experience, efforts to improve web accessibility in private industry are often not prioritized by business stakeholders $[1,2]$. Whereas search engine optimization (SEO) also plays a role in user experience [3], advocacy for SEO is more easily received by stakeholders for its contribution to meeting business goals by way of increasing brand visibility. While the general assumption here is that web accessibility benefits only a small proportion of consumers for most businesses, the social and ethical impacts of web accessibility advocacy are outcomes that will ultimately benefit companies and organizations [4].

As the implications of accessibility extend far and wide, we performed a study on hidden codes involved in web accessibility with the intention to describe an approach to accessibility audits in private industry. While our research remains ongoing, we have found valuable insights into web accessibility and its correlation to increased SEO, better coding practices, and, ultimately, greater accountability, advocacy, and coalition towards more inclusive user experiences. We believe such research will entice stakeholders to recognize the value of web accessibility and the benefits it holds for both business and consumers.

In sum, this case study aims to advocate for SEO and web accessibility for better user experience by:

\section{METHODS}

To achieve our goals, we adopted both automatic and manual testing methods. HRsimple's blog page [5] was selected for testing given that it receives the highest amount of new-user web traffic based on the site's Google Analytics. Six experts (Mexperience $=3$ yrs, $\mathrm{SD}=3.61, \mathrm{MAX}=10 \mathrm{yrs}, \mathrm{MIN}=1 \mathrm{yr}$ ) were invited to evaluate the latest version of HRsimple's blog site: two experts have more than 5 years of experience and four experts have less than 3 years of experience in UX design and web development.

Our first round of iterative testing focused on addressing HRsimple's SEO performance. An automated diagnosis of the site's SEO was conducted using Google Chrome's Lighthouse developer tool. To measure the efficacy of our methods, we set the benchmark for successful improvement at an SEO score of 97 or greater. The second round of testing comprised formative accessibility audits. Automatic testing was conducted using Google Chrome extensions 
(e.g., ARC by TPGi [6] and WAVE [7]). Manual testing was conducted by keyboard accessibility and structural semantic HTML, ARIA-screen reader functionality (e.g., Chrome Screen Reader [8], NVDA [9], and web developer tools/inspector). The accessibility audits targeted over $95 \%$ of satisfaction rate (i.e. evaluated as 'pass' for the success criteria). A summative assessment was then conducted by an additional three experts to measure the effectiveness of the revision. The iterative testing process spanned a total of 12 weeks, with phases including: 1) diagnosis of the UX issues, 2) design solution workshop, 3) prototyping, 4) implementation of revised code in the development framework, and 5) documentation and communication with internal/external team members.

\section{FINDINGS \& RESULTS}

\subsection{Search Engine Optimization (SEO)}

The initial SEO audit of HRsimple's blog page by Chrome Lighthouse revealed a score of $85 / 100$. The issues negatively impacting SEO score were: 1) uncrawlable links (i.e. difficult for search engines to understand what contents are being linked to); 2) unrecognizable alt-text (i.e. non-descriptive text and long file names); 3) generic button labels (i.e. difficult for search engines to understand the functionality/purpose of a button). Uncrawlable links on the blog page were found in its original pagination system. As pagination was linked numerically (i.e. "1", "2", "3"...), each page link was deemed uncrawlable because the number alone did not describe what contents would be found through that link. As a solution, the pagination was replaced with a "Next Page" link that provided a clearer indication of additional blog entries accessible through that interaction. To address issues with alt-text, decorative images across the blog page were attributed with null alt-text (alt=""), an SEO best practice that benefits web accessibility efforts as well. Generic button labels (i.e. "Learn More") were replaced with more semantically descriptive labels (i.e. "Get Product Info"), which resolved the issue of ambiguous context. These revisions effectively increased the blog page's SEO score to 100 , and were thus communicated with HRsimple's internal content writers and marketers to implement as guidelines for content management. As a result, HRsimple's blog site is listed in a higher position among search results, and site visitors seemed to stay longer (based on Google Search Console (GSC) data, please see Discussion for the details). Thus, SEO was easily improved upon by reorganizing the blog page's metadata and semantic structure with schema [10], elements that may not be visible in terms of interface design, but surely contribute to holistic user experience and overlap with efforts in web accessibility.

\subsection{Accessibility}

Initial results from automatic testing conducted using WAVE and ARC tools uncovered several accessibility issues: 1) missing bypass blocks from the tab order navigation, 2) insufficient use of landmarks (i.e. navs nested in navs; pages marked only with 'main'), 3) missing/mislabeled ARIA attributes, and 4) insufficient color contrast across buttons and hyperlinks. Our in-depth manual testing confirmed these major issues, in addition to issues regarding: keyboard-operated focus, tab order, alt-text, screen reader interactions for images, meaningful sequence related to header tags, and undistinguished hyperlinks. For the scope of this paper, we will review the focus feature of keyboard-operated tabbing.

We found that keyboard-operated focus items were not always clearly visible, especially on links and buttons with additional styling. HRsimple's website initially used Bootstrap v4.0 [11], which was not compatible with color contrast and presented a focus that did not adhere to tabbing and visibility standards set by the Web Content Accessibility Guidelines (WCAG) [12]. Figures F1 and F2 display the visual definition of keyboard tabbing focus on a red "Sign up" button.

Normally, the default focus tab of major web browsers has a thick, blue line around a focusable item. To override this default outline and adhere to HRsimple's branding guidelines, we added additional code to the CSS file. Mozilla Development Network (MDN) Web Docs [13] recommends changing the "outline-color" CSS variable to modify the default blue color with contrast ratio 4.5:1 (Safari required changing the "outline" variable, as opposed to "outline-color" used in other browsers). However, we discovered that overriding the default outline for focus items comes with accessibility concerns. Figures F3 and F4 denote the example CSS used to modify the focus of Bootstrap's class .btn-danger:

Figure F3 (Before)

.btn-danger: focus \{

color: \#FFFFFF;

background-color: \#C82333;

box-shadow: $0,0,0,0.2 \mathrm{rem}$ rbga $(225,83,97,0.5)$; \}

The additional CSS code in Figure F4 (below) shows how we communicate the focus of keyboard-operated tabbing to users with hidden-code:

Figure F4 (After)

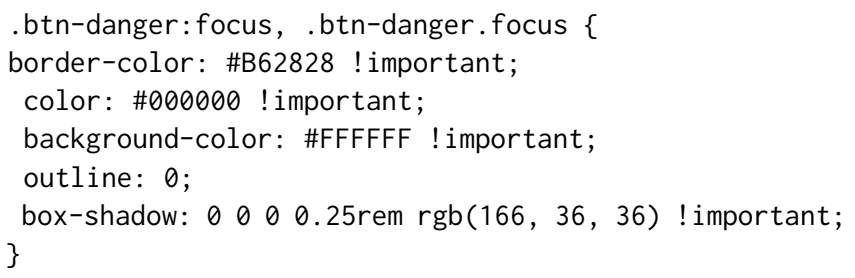

The most significant CSS style for button focus is "outline: 0 ;" and "box-shadow: 000 0.25rem rgb(166, 36, 36) !important;", as it modifies the default focus that originally employs a thin blue line. However, with CSS frameworks like Bootstrap, the focus changes significantly. As mentioned earlier in our case, the focus on buttons, especially Bootstrap's class .btn-danger and .btn-warning, becomes indistinguishable from other non-focused items.

The foreground of the outline color, $\# 8 \mathrm{C} 1 \mathrm{~F} 1 \mathrm{~F}$, in contrast with the background color of HRsimple's website, \#FFFFFF, complies with WCAG AA and AAA standards at 9:1 contrast ratio. For the background color, \#FFFFFF, and the focused text color, \#000000, contrast ratio is $21: 1$. These are just one example of the many items analyzed in our research.

We applied the same, rigorous testing to links, search forms, and other features across HRsimple's blog page. By manually tabbing through the website, we were able to identify usability issues not detected by automated tools, including better understanding of how a keyboard user experiences a digital environment. Overall, 


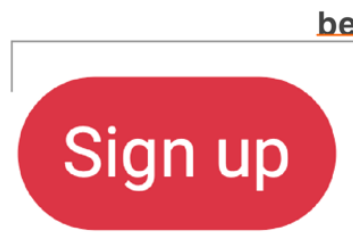

F1 before

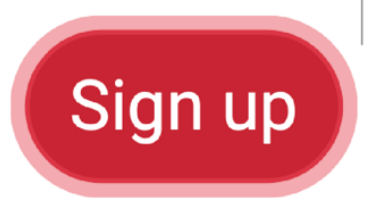

F1.2

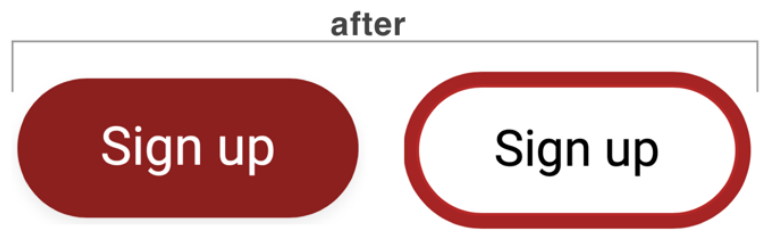

F2
F2.2

Figure 1: and F2: When focused, the initial "Sign up" button displayed a subtle indication via a blurred red border (Fig. F1). Our solution for this example was to change the button color, the color of the text, and the outline of the button when focused to increase visibility. Figure F2 shows the outcome of these changes made to the button's CSS code.

Table 1: GSC IMPRESSIONS Per Day

\begin{tabular}{|c|c|c|c|c|}
\hline Device & $\begin{array}{l}\text { Feb. } 82021 \text { to April } 22 \\
2021 \text { ( } 75 \text { days) }\end{array}$ & $\begin{array}{l}\text { April } 232021 \text { to June } 29 \\
2021 \text { ( } 68 \text { days) }\end{array}$ & Change & Percent Change \\
\hline Desktop & $6,828.71$ & $8,336.72$ & $1,508.01$ & $122.08 \%$ \\
\hline Mobile & $3,683.81$ & $9,932.79$ & $6,248.98$ & $269.63 \%$ \\
\hline Tablet & 92.76 & 150.69 & 57.93 & $162.45 \%$ \\
\hline Total & $10,605.28$ & $18,420.2$ & $7,814.92$ & $173.69 \%$ \\
\hline Average & 3535.09 & $6,140.07$ & $2,604.96$ & \\
\hline
\end{tabular}

we learned how to write better code that effectively communicated not only with our intended audience, but also with users of screen readers by including "aria-labelledby" or "aria-label" in place of the label tag. Where applicable, we used label tags to identify properties with ids and hid those labels visually if they did not hold merit for visual users; if a label was not feasible, we used "aria-labelledby" or "aria-label".

While this paper is narrow in scope, we believe the focus feature for keyboard users was a significant element to analyze. After upgrading the website to Bootstrap beta v5.0 [14], the color contrast adhered to industry standards, but still Bootstrap's default focus for tabbing needed improvement. Additionally, automated accessibility tools such as ARC and WAVE did not flag the focus on links and buttons with additional style such as Bootstrap buttons. This problem lends to a keyboard operator or screen reader losing the tab location quite easily.

\section{DISCUSSION}

HRsimple's website is seeing positive results related to Google Search Console (GSC) [15] "Impressions" and "Position". From what we understand about GSC information, "Impressions" is how many times a website appears in a search results and is seen by a user. "Position" is the position found in the search results. As of February 8 , 2021, hrsimple.com started tracking information from GSC. From this date until April 22, 2021, hrsimple.com Impressions averaged 6,829 for Desktop, 3,684 for Mobile and 93 for Tablet users per day. For the Position, hrsimple.com averaged 31 for Desktop, 19 for Mobile, and 29 for Tablet users per day.

Comparing this information to our research update that occurred from April 23 to June 29, 2021, hrsimple.com Impression rate averaged 8,337 for Desktop, 9,933 for Mobile and 151 for Tablet users per day. This displays a change in Desktop Impressions by 1,508 per day ( $122 \%$ increase); Mobile by 6,248 (269\% increase); Tablet by 58 (162\% increase). See 1 for a clearer visualization of Impressions numbers. As for the Position from February 8 to April 22, 2021, Desktop averaged 31; Mobile 19; and Tablet 29. The Position after April 22, 2021 averaged 25.1 for Desktop; 9.3 for Mobile; and 10.8 for Tablet. See 2 for a clearer visualization of Position numbers. Overall, the Impressions from GSC changed from 795,396 (February 8, 2021 to April 22, 2021) to 1,252,574 (April 23, 2021 to June 29, 2021), a 457,178 (157\%) change. Also, the overall "Position" moved down from 26.33 to 15.06 in the same timeframe as described above.

While our data is still small and needs more time to show conclusive and meaningful results, the stakeholders of hrsimple.com have begun to see higher-quality web traffic since we performed this audit and submitted the code to production, on April 23, 2021. Theoretically, we believe that our contributions to web accessibility enable us to not only write better code, comply with WCAG AA and AAA standards, but web accessibility ultimately increased the company's SEO competition, especially for mobile users.

\section{REFLECTION}

The discussion section describes recent information from GSC, but this information is not to say that our research and web accessibility adjustments are a significant contributor to Impressions and Positions. One of the possible outcomes from this information is that the labor market and economy are beginning to improve, which, in turn, could posit that more people are searching online for employment law information (the services hrsimple.com offers). As stated above, our data is still small and demands more time to settle in and adjust. However, if we continue seeing a positive increase over the next few months, we will report this information and generate a specific correlation to web-accessibility and SEO with GSC data. 
Table 2: GSC POSITION

\begin{tabular}{|c|c|c|c|c|}
\hline Device & $\begin{array}{l}\text { Feb. } 82021 \text { to April } 22 \\
2021 \text { ( } 75 \text { days) }\end{array}$ & $\begin{array}{l}\text { April } 232021 \text { to June } 29 \\
2021 \text { (68 days) }\end{array}$ & Change & Percent Change \\
\hline Desktop & 31 & 25.1 & 5.9 & $93.55 \%$ \\
\hline Mobile & 19 & 9.3 & 9.7 & $78.95 \%$ \\
\hline Tablet & 29 & 10.8 & 18.2 & $65.52 \%$ \\
\hline Total & 79 & 45.2 & 33.8 & $79.75 \%$ \\
\hline Average & 26.33 & 15.06 & 11.27 & \\
\hline
\end{tabular}

\section{REFERENCES}

[1] Horton, Sarah, and David Sloan. 2017. Accessibility for business and pleasure. Interactions 23, 1, 80-84.

[2] Kalbag, Laura. 2017. Accessibility for everyone. A Book Apart

[3] Amerland, David. 2013. Google semantic search: Search Engine Optimization (SEO) techniques that get your company more traffic, increase brand impact, and amplify your online presence. Que Publishing.

[4] World Wide Web Consortium (W3C). Web Accessibility is an Aspect of Corporate Social Responsibility (CSR). Retrieved from https://www.w3.org/WAI/businesscase/archive/soc\#: :text=Web\%20accessibility\%20can\%20impact\%20an, commitment $\% 20$ to\%20providing\%20equal\%20opportunities.

[5] hrsimple blogs. Retrieved from https://www.hrsimple.com/blog

[6] TPGi. ARC toolkit. Retrieved from https://www.tpgi.com/arc-platform/arctoolkit/
[7] WebAIM. Web Accessibility Evaluation Tool (WAVE). Retrieved from https:// wave.webaim.org/

[8] Google. Chrome Screen Reader. Retrieved from https://chrome.google.com/ webstore/detail/screen-reader/kgejglhpjiefppelpmljglcjbhoiplfn?hl=en

[9] NV Access. NVDA. Retrieved from https://www.nvaccess.org/

[10] Schema.org. Welcome to Schema.org. Retrieved from https://schema.org/

[11] Bootstrap 4.0. Getting Started. Retrieved from https://getbootstrap.com/docs/4.0/ getting-started/introduction/

[12] W3C. Web Content Accessibility Guidelines (WCAG) Overview. Retrieved from https://www.w3.org/WAI/standards-guidelines/wcag/

[13] Mozilla Developer Network (MDN). Accessibility. Retrieved from https:// developer.mozilla.org/en-US/docs/Web/Accessibility

[14] Bootstrap 5.0. Getting Started. Retrieved from https://getbootstrap.com/docs/5.0/ getting-started/introduction/

[15] Google. Search Engine Console. Retrieved from https://search.google.com/searchconsole/about 